\title{
Spectral EEG Features of a Short Psycho-physiological Relaxation
}

\author{
Michal Teplan ${ }^{1}$, Anna Krakovská $^{1}$ and Marián Špajdel ${ }^{2,3}$ \\ ${ }^{1}$ Institute of Measurement Science, Slovak Academy of Sciences, Bratislava, Slovakia, michal.teplan@ savba.sk \\ ${ }^{2}$ Institute of Normal and Pathological Physiology, Slovak Academy of Sciences, Bratislava, Slovakia \\ ${ }^{3}$ Faculty of Philosophy and Arts, University of Trnava, Slovakia
}

\begin{abstract}
Short-lasting psycho-physiological relaxation was investigated through an analysis of its bipolar electroencephalographic (EEG) characteristics. In 8 subjects, 6-channel EEG data of 3-minute duration were recorded during 88 relaxation sessions. Time course of spectral EEG features was examined. Alpha powers were decreasing during resting conditions of 3-minute sessions in lying position with eyes closed. This was followed by a decrease of total power in centro-parietal cortex regions and an increase of beta power in fronto-central areas. Represented by EEG coherences the interhemispheric communication between the parieto-occipital regions was enhanced within a frequency range of $2-10 \mathrm{~Hz}$. In order to discern between higher and lower levels of relaxation distinguished according to self-rated satisfaction, EEG features were assessed and discriminating parameters were identified. Successful relaxation was determined mainly by the presence of decreased delta-1 power across the cortex. Potential applications for these findings include the clinical, pharmacological, and stress management fields.
\end{abstract}

Keywords: EEG, relaxation, biosignal analysis, coherences.

\section{INTRODUCTION}

$\mathrm{T}$ HE RELAXATION response is an integrated body reaction that reflects the voluntary resting state of both the body and the mind. The characterization of the changes in brain activity during the resting state is relevant to many fields, including stress reduction, sleep deprivation, and the testing of pharmacological substances related to drugs with hypnotic and sedative effects. Relaxation response influences mental and physical health and has an important role in moderating tension and psychosocial stress. In a broader sense, stress is acknowledged as one of the major problems of modern society. Regular relaxation practice can affect the various physiological and psychological parameters involved in aging, digestion, general well-being, and psycho-somatic disease. Consequently, there is a growing need to monitor the physiological processes associated with relaxation and stress response.

The term "relaxation" conveys positive and beneficial phenomena. However, it is difficult to define psychophysiological relaxation in terms of specific physiological parameters. According to Travis [1], five categories of physiological variables have the potential to discern between the levels of relaxation, including breath and heart rates, as well as skin conductance. Similar parameters for the characterization of stress levels were reported by Vavrinský [2]. Physiological indicators of the relaxation response introduced in Foster [3] include decreased oxygen consumption, respiration, and heart rate, in addition to increased alpha brain wave production. According to Ossebaard [4], relaxation is comprised of biological, psychological, and social components.

Another useful source of information on relaxation is research on meditation. In fact there are no issues focused directly on EEG dynamics during relaxation induction in the literature. Differences between two distinct states or conditions are usually investigated, e.g., before the state of relaxation or meditation and during or after such a state. However, here we focused on a gradual change of the physiological state. Various types of meditation are known to induce a relaxed state $[5,6,1]$. Usually, with increased level of meditation, a deeper state of relaxation can be established. This finding is supported in parallel by subjective observations and physiological parameters (e.g., respiratory rate, skin conductance, and production of plasma lactate) [1].

However, the evidence presented in the literature is inconsistent regarding the characterization of relaxation using specific EEG features. The increased power of the alpha and theta frequencies $[5,7,8,3,9]$ and interhemispheric synchronization, especially frontal alpha coherence [1], are usually considered to be neurophysiological indicators of a state of sensorimotor and mental rest.

In our previous study, we observed an increase in the alpha and theta powers and an enhanced frontal alpha coherence [10] after two months of audio-visual stimulation training (25 repetitions of 30-minute intervals of relaxation) [11].

The aim of the present study was to directly examine the spectral EEG characteristics during a 3-minute interval while the subjects were in a resting state. In particular, two sequential tasks were addressed: 1) the detection of changes in spectral EEG measures during 3-minute general sensorimotor relaxation and 2) the selection of EEG features that are able to distinguish between self-rated successful relaxation and relaxation which was subjectively perceived as less efficient.

\section{MATERIAL AND METHODS}

\subsection{EEG recording}

Eight right-handed, healthy subjects ( 3 females and 5 males, mean age 27 years, SD 7 years) volunteered for the experiment. The subjects were instructed to lie down comfortably 


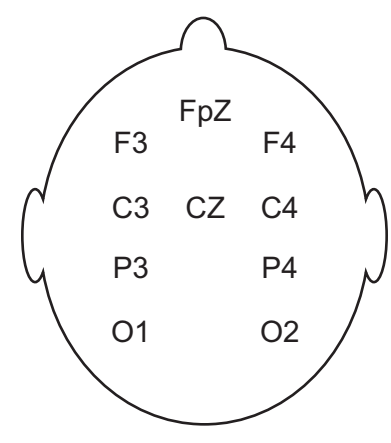

Fig. 1: The EEG montage used in this study is shown. The active electrodes were placed at the F3, F4, C3, C4, P3, P4, O1, and O2 locations with the reference and ground electrodes at the $\mathrm{Cz}$ and $\mathrm{Fpz}$ points, respectively.

on their backs, keeping their eyes closed and relax both physically and mentally. No further instructions or technique were provided regarding how to establish a relaxing state. The experiment was conducted in a darkened, electrically shielded room. The subjects placed in these conditions were expected to realize a state of sensory, motor, as well as mental relaxation.

The EEG data for a 3-minute interval were recorded from eight scalp positions according to the International 10-20 system: F3, F4, C3, C4, P3, P4, O1, and O2 (Figure 1). The reference electrode was located at $\mathrm{Cz}$ and the ground electrode at Fpz. The $\mathrm{Ag} / \mathrm{AgCl}$ electrodes were placed on a standard EEG cap (Electro Cap Inc.). Impedances were kept below $5 k \Omega$ and balanced within $1 k \Omega$. The parameters for our prototype EEG recording unit were as follows: amplifying gain: 402; sampling frequency: $500 \mathrm{~Hz}$; A/D converter resolution: 14 bits; input resolution: $0.46 \mu \mathrm{V}$; noise: $\max 4.1 \mu \mathrm{V}$ pp. (0.07 to $234 \mathrm{~Hz})$; low pass filter: $234 \mathrm{~Hz}(-3 \mathrm{~dB})$; and high pass filter: $0.07 \mathrm{~Hz}(-3 \mathrm{~dB})$.

From the 8 signals recorded from active electrodes, six bipolar signals (F3-C3, F4-C4, C3-P3, C4-P4, P3-O1, and $\mathrm{P} 4-\mathrm{O} 2$ ) were derived off-line in order to avoid the undesirable effects of the common reference electrode. All of the signals were visually inspected; the sequences containing artefacts or sleep phenomena were excluded with support of information from forms filled in by participants. Preprocessing was completed by the application of a digital high pass FIR filter with a cut-off frequency of $0.5 \mathrm{~Hz}$ and a 3000-point Blackman window.

\subsection{Measures}

We analyzed the total power, relative power, and magnitude squared coherence in nine frequency bands: delta-1 (0.5-2 $\mathrm{Hz})$, delta-2 $(2-4 \mathrm{~Hz})$, theta-1 (4-6 Hz), theta-2 $(6-8 \mathrm{~Hz})$, alpha-1 (8-10 Hz), alpha-2 $(10-12 \mathrm{~Hz})$, beta-1 $(12-16 \mathrm{~Hz})$, beta-2 $(16-30 \mathrm{~Hz})$, and gamma $(30-45 \mathrm{~Hz})$. The total power ranged from 0.5 to $45 \mathrm{~Hz}$.

The frequency analysis was calculated using a fast Fourier transform within a 2 -second window with 0.75 seconds of overlap. All of the calculations were carried out in the Matlab environment (by Mathworks, version R2008b) with the use of the pwelch and cpsd functions. The powers were analyzed in 6 cortical locations and were obtained by the offline data transformation into bipolar mode as described above. The coherences were investigated between 11 different pairs (F3C3-F4C4, etc.) of the 6 scalp regions. Combinations with common electrodes (e.g., F3C3-C3P3) were not considered in order to avoid spurious results caused by contribution of the common electrode into both signals.

Each subject participated in 24 recording sessions and attended only one session per working day. The data were collected over a 2-month period. Prior to participation, every subject completed an entrance form assessing general health status, medication, involvement, motivations and expectations of the experiment. One of the eight participants reported prior experience in meditation, yoga or other relaxation techniques. Before each session, participants filled out a one-page structured form where their actual overall status was reported in a form of health, emotional and mental components. Also their medication and caffeine or nicotine intake was questioned. The subjects were asked to rate the progress in relaxation attained during the 3-minute resting period after each recording session. A seven-point bipolar Likert scale [12] was used for assessment. The relaxation extremes rated by the scale ranged from "tense or aroused" ( -3$)$ to "relaxed" (+3).

The time course of the various EEG measures (powers and coherences) during each 3-minute interval was determined based on the spectra, which were computed in 2-second sub windows. The resulting curves were fitted using linear regression. The magnitude of the time trends was evaluated by F-test values that were obtained from testing whether the regression slope differs from the zero one.

\section{RESULTS}

\subsection{General relaxation}

In this part we examined all EEG data that were measured, regardless of subjects' self-rated satisfaction. 11 artefact-free recordings from each participant were selected. For each measure and cortex location, we had a total of 88 time curves from the 8 subjects. For most of the EEG measures, a linear time course was observed during the 3-minute recording period. In order to identify the prominent EEG features that characterize the relaxation process, we focused on the strongest time trends. All time trends were quantified by F-test values derived from evaluation of differences of the regression slopes from the zero slope. The F-values were modified with addition of sign designating decreasing (minus) or increasing (plus) trends. Modified F values were not distributed normally. Therefore, we used nonparametric testing to identify changes in the EEG powers during resting conditions. A sign test was applied to test whether medians differed from zero.

The time courses are illustrated as average time curves $(n=88)$ to highlight the measures of the most significant changes within the sessions. A decrease in total EEG power 


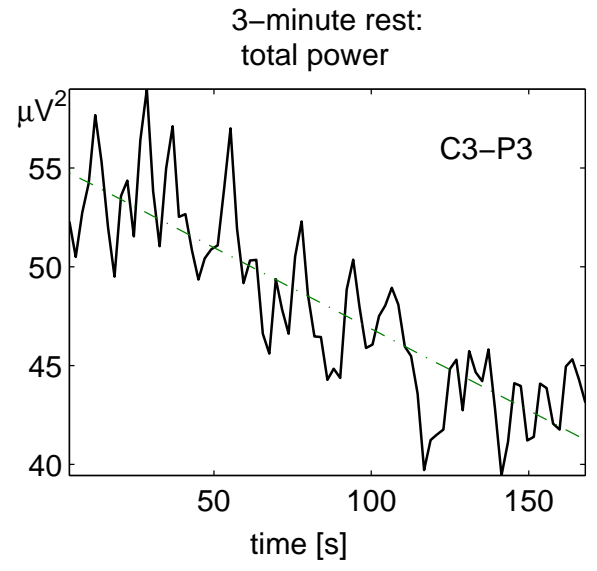

Fig. 2: The total EEG power changes in the C3-P3 location during general relaxation are shown for the 8 subjects. The grand average time course, which was calculated by averaging the 88 curves from each of the recording sessions, is presented.

was observed in the centro-parietal location C3-P3 (Figure 2) and C4-P4 (p-values $<0.02$ and 0.05 , respectively).

The strongest decrease was related to the alpha-1 wave contribution. With the exception of the fronto-central areas, the relative alpha-1 power dropped in all of the locations: C3-P3, C4-P4, P3-O1, and P4-O2 (all p-values $<10^{-5}$, Figure 3). The most significant increase was found for the beta- 1 relative power in the fronto-central F3-C3 (Figure 4) and F4$\mathrm{C} 4$ areas (p-values $<10^{-5}$ and $10^{-3}$, respectively). Moreover, the higher frequency bands, beta-2 and gamma, also increased their expression in the F3-C3 area (p-values $<0.008$ and 0.004, respectively; Figure 4).

The most prominent EEG effects were realized within the alpha-1 waves in the P4-O2 area (Figure 3); the average relative alpha- 1 power was diminished by $30 \%$ of its initial value. This measure decreased in $76 \%$ of all the single measurement sessions. The decrease of the relative alpha-1 power often led to positive relative increments for all the other bands. This fact is evident in the bar graphs shown in Figures 3 and 4, which compare the spectral band characteristics in a form of grand averages from the initial and the final 20-second intervals of the 3-minute recording period.

Significant changes were also identified in the coherence data. The interhemispheric communication between the parieto-occipital centres was enhanced. This conclusion was based on the increase in the P3O1-P4O2 coherence in the four neighbouring power bands, which include the delta-2, theta-1 (Figure 5), theta-2, and alpha-1 (all p-values $<0.008$ ).

\subsection{Successful relaxation}

Our second goal was to determine which of the EEG measures are associated with self-rated successful relaxation. Therefore, the recordings from the previous section used for specifying the state of general relaxation were divided here into
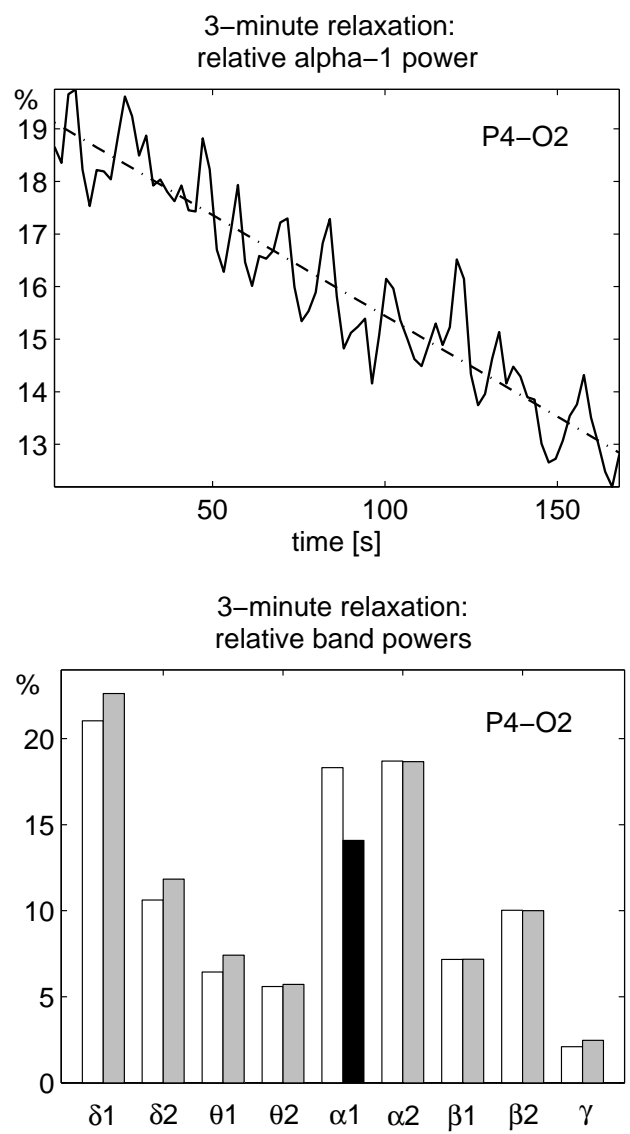

Fig. 3: Above: The time course for the grand average alpha-1 relative band power in the $\mathrm{P} 4-\mathrm{O} 2$ location during general relaxation. Bellow: The grand average relative band powers. The data for the initial (white) and closing periods (gray and black) are presented for the P4-O2 cortex location. Significant changes in the alpha-1 band are highlighted in black.

two sets according to the subjects' self-ratings of the relaxation progress. Out of 11 recordings from each subject the 4 records with the highest ratings and the 4 records with the lowest ratings of attained relaxation level were chosen. The subjects differed in their strategies for the relaxation level ratings. Some of the subjects reported extreme values (-3 or 3$)$, while some reported only values closer to zero $(-1,0,1)$. The rating distribution was asymmetrical with the positive ratings occurring more frequently. The mean rating values were -0.06 for less successful relaxation and 1.88 for more successful relaxation.

For all of the participants, the linear regression was calculated separately for each of the four recordings given his or her extreme low or high relaxation self-rating. We focused on a comparison of the two groups according to the following three parameters derived from the time course of the single EEG spectral characteristics. Two of them were the initial and final values obtained from linear regression of the time course accounted for the first and last 6 seconds of the recordings, re- 

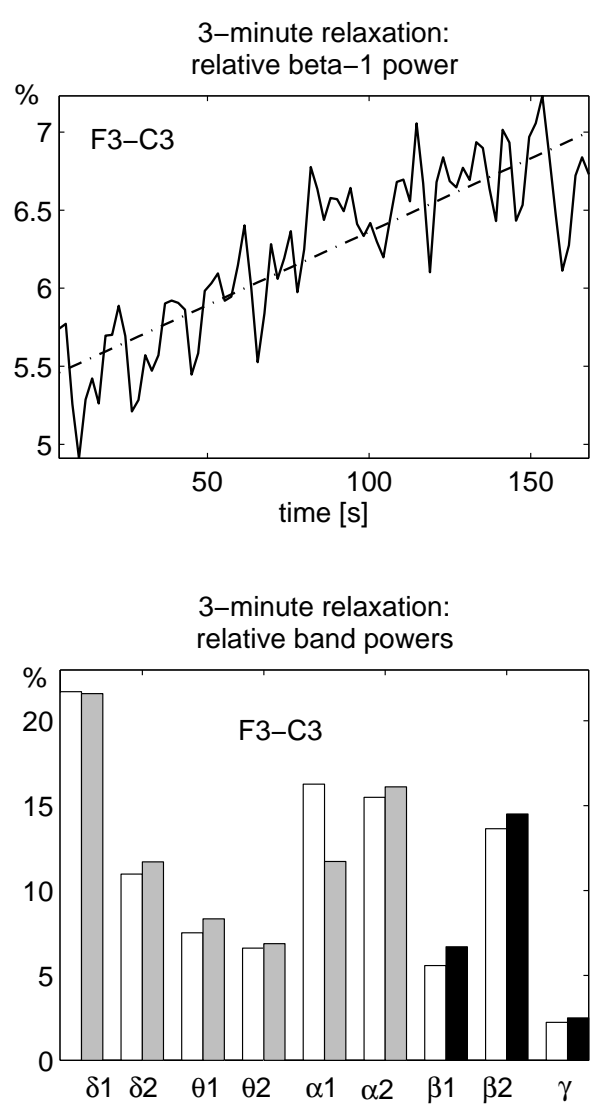

Fig. 4: Above: The time course for the grand average beta-1 relative band power in the F3-C3 location during general relaxation is shown. Bellow: The grand average relative band powers are shown. The initial (white) and closing periods (gray and black) in the F3-C3 cortex location are depicted. The bands with significant changes are highlighted in black.

spectively. The slopes were represented by the sign modified F-values introduced in the previous section. In order to find the distinct features of the two relaxation categories, a nonparametric Kruskall-Wallis test was used for the 2 groups (n $=32 ; 4$ sessions for each of the 8 subjects). The significance threshold was $p<0.05$.

Deeper relaxation was associated with lower final values of the slowest spectral components. Lower contribution of delta-1 waves was present in the following derivations: F4C4, C3-P3, and P3-O1 (all p-values $<0.03$, Figures 6 and 7). In the three remaining cortex locations, the delta-1 shift for successful relaxation was lower as well, however, not statistically significant. Already at the beginning of the relaxation sessions, the beta-2 power in the P3-O1 location was higher for the more successful relaxation sessions than for the less successful relaxation sessions $(p<0.04)$.

For certain coherences, lower final values were obtained interhemispherically. Specifically, decrement was found for the better relaxation in case of the alpha- 1 coherence (between the $\mathrm{C} 3 \mathrm{P} 3$ and $\mathrm{P} 4 \mathrm{O} 2$ locations, $p<0.04)$ and the coherence

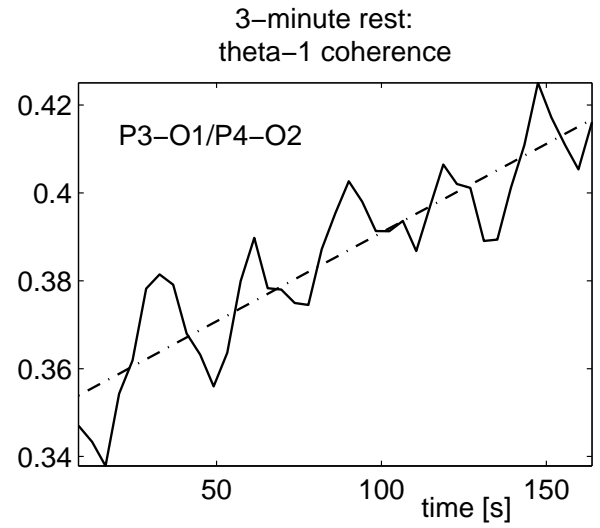

Fig. 5: The time course of grand average for theta-1 coherence between the P3-O1 and P4-O2 locations during general relaxation is shown. The mean time course was calculated by averaging the 88 curves from each of the recording sessions.

in the beta-1 band (between the F4C4 and C3P3 locations, $p=0.017)$. Different relaxation states displayed also different regression slopes according to the modified F-values. For more successful relaxation, rise of the alpha-2 coherence between the $\mathrm{F} 3 \mathrm{C} 3$ and P3O1 locations ( $p<0.002$, Figure 6) and decrease of the coherence in the beta- 1 range between the C3P3 and C4P4 locations $(p<0.05)$ was observed.

\section{DISCUSSION}

A decrease in the total power across the entire cortex during the relaxation condition implies that, overall, the subjects' brain activity gradually diminished along the relaxation process. We anticipated an increase in the alpha powers $[3,7,8]$; but surprisingly, we discovered a pronounced and significant decrease in alpha-1 power, which was a definite leading factor in the observed decline in overall performance (Figure 3 ).

In the literature, it is a commonly accepted fact that the increase in the alpha waves is associated with rest and relaxation [3]. However, this phenomenon may be partly related to closing the eyes. Closing the eyes usually initiates rest states, which can occur for short periods of time, even when the subject is sitting or standing. In our study, the relative power of the alpha- 2 band was increased for all of the cortex regions, but only during the first 30 seconds. This increase was followed by a strong decline in the power of the alpha- 1 waves for the remainder of the 3-minute relaxation period.

However, a decrease in the relative alpha power did not guarantee that successful relaxation was achieved. Self-rated satisfaction with a relaxation state correlated with the decreased expression of the delta-1 relative power.

Indicators of enhanced relaxation in a form of lower contribution of the slowest waves remove any speculations about the correlation of the observed relaxation states with periods with eventual occurrence of sleep onset. Sleeping states are characterized instead by the increased occurrence of delta waves. 

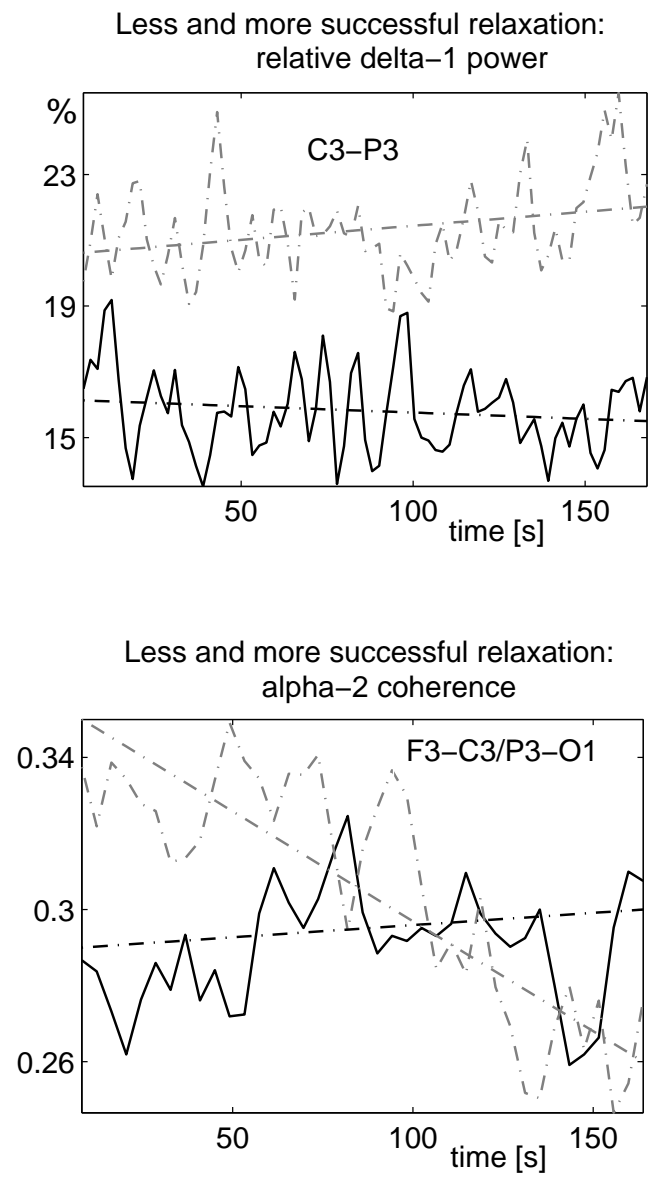

Fig. 6: The difference between less (gray) and more (black) successful relaxation is illustrated. The grand average of time course was calculated by averaging all of the curves for each of the recording sessions. The difference in the final values for relative delta-1 power in C3-P3 (above) and in the slopes for alpha-2 coherence between F3-C3 and P3-O1 (bellow) are shown.

During the general relaxation process we observed an increase in the coherences between the hemispheres at the parieto-occipital sites in the $2-10 \mathrm{~Hz}$ frequency range and a decrease in some of the beta- 1 coherences interhemispherically. However, it is not possible to make a clear conclusion regarding the ability of coherence to discern between the more and less successful relaxation states.

This conclusion is in accordance with the findings of Gaylord [13], who reported that the coherence was higher in the frontal and central areas only during transcendental meditation practice relative to the closed eyes condition, but not during progressive muscle relaxation.

Several of the subjects occasionally reported some form of thinking (often related to their work or everyday problems). It can be assumed that internal thought would occur regularly for most of the participants, regardless of their awareness of it, as the subjects had no special training in controlling their mental activity during the relaxation. This finding is in ac-

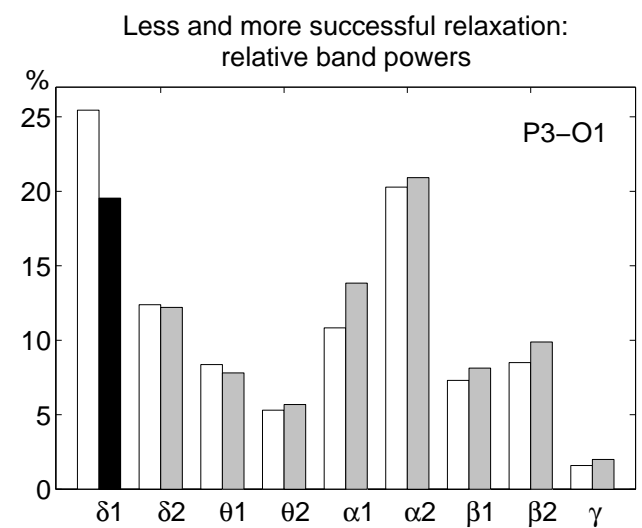

Fig. 7: A comparison of the grand average relative band powers at the end of the 3-minute relaxation period in the P3-O1 location are shown. Less successful relaxation (white) and more successful relaxation (grey and black). Black represents statistically significant difference.

cordance with the data from questionnaires in which the participants occasionally reported their thought processes. Regarding this fact, our aim was not to study relaxation under restricted conditions; for example, meditation studies focus on highly trained subjects. In contrast, our goal was to investigate the relaxation processes in normal adult population. For most individuals, pleasant and beneficial relaxation can involve episodes with relaxed thought processes. Hence, we instructed the participants to relax generally, which includes physical, mental, and emotional aspects, and we did not provide any special instructions or methods specifying how to relax.

Upon completion of this experiment, half of the participants reported their capacity for relaxation to be improved due to repetitive participation in the relaxation experiment sessions.

We have introduced a new approach to the investigation of relaxation through time course of EEG features. Furthermore, we have confronted general assertions on relaxation with the computed spectral EEG characteristics. In this sense, our results contribute to the characterization of relaxation processes with the use of spectral EEG features and might be beneficial for further research in areas like neurofeedback and resting states analysis. In the case of neurofeedback, the features found to be characteristic for the state of relaxation might be used in biofeedback training protocol in order to improve relaxation induction.

Moreover, we have shown that with the use of EEG data it is possible to discriminate between two relaxation categories formed according to the quality of relaxation. We have found that the focus should be directed to the slowest brain waves. It is important to note that our results may be specific to the conditions of our study; therefore, different EEG outcomes may result from a different set of relaxation conditions. Another 
factor influencing outcomes of the study might be a choice of unipolar or bipolar EEG due to data processing.

For future studies we suggest simultaneous monitoring of additional physiological variables (e.g., cardiovascular, respiratory, muscular, skin conductance, and blood parameters responsive to the level of stress) so that the EEG parameters can be associated with complex physiological responses more directly. Also a more prolonged relaxation period could be investigated, and subjects' mental processes could be monitored by more specific questionnaires.

\section{ACKNOWLEDGEMENT}

The research was supported by the grant VEGA 2/0043/13.

\section{REFERENCES}

[1] Travis, F. (2001). Autonomic and EEG patterns distinguish transcending from other experiences during Transcendental meditation. International Journal of Psychophysiology 42, 1-9.

[2] Vavrinský, E. (2005). Thin-film microelectrodes for electro-chemical conductive sensors applied in biomedical monitoring of stress. Ph.D. thesis, Slovak Technical University in Bratislava, Faculty of Electrical Engineering and Informatics, Department of microelectronics.

[3] Foster, D. (1990). EEG and subjective correlates of alpha-frequency binaural-beat stimulation combined with alpha biofeedback. Memphis State University.

[4] Ossebaard, H. (2000). Stress reduction by technology? An experimental study into the effects of brainmachines on burnout and state anxiety. Applied Psychophysiology and Biofeedback 25, 93-101.
[5] Banquet, J. (1973). Spectral analysis of the EEG in meditation. Electroencephalography and Clinical Neurophysiology 35, 143-151.

[6] Aftanas, L., Golocheikine, S. (2001). Human anterior and frontal midline theta and lower alpha reflect emotionally positive state and internalized attention: highresolution EEG investigation of meditation. Neuroscience Letters 310, 57-60.

[7] Lindsley, D. (1952). Psychological phenomena and the electroencephalogram. Electroencephalography and Clinical Neurophysiology 4, 443-456.

[8] Brown, B. (1970). Recognition of aspects of consciousness through association with EEG alpha activity represented by a light signal. Psychophysiology 6, 442-452.

[9] Jacobs, G., Friedman, R. (2004). EEG Spectral Analysis of Relaxation Techniques. Applied Psychophysiology and Biofeedback 29, 245-254.

[10] Teplan, M., et al. (2006). EEG responses to long-term audio-visual stimulation. International Journal of Psychophysiology 59, 81-90.

[11] Teplan, M., et al. (2011). Direct and transient effects of audio-visual stimulation on EEG. Computer Methods and Programs in Biomedicine 102, 17-24.

[12] Likert, R. (1932). A Technique for the Measurement of Attitudes. Archives of Psychology 140, 1-55.

[13] Gaylord, C., et al. (1989). The effects of the transcendental meditation technique and progressive muscle relaxation on EEG coherence, stress reactivity, and mental health in black adults. International Journal of Neuroscience 46, 77-86. 